\title{
Social eavesdropping by stingless bees
}

\section{H. Richards ${ }^{1}$}

Published online: 29 July 2021

(c) International Union for the Study of Social Insects (IUSSI) 2021

In our electronically connected age, many people carry cell phones that accurately track their movements, eavesdrop on their conversations, and record many types of behavioural preferences. Collection of public information by observers who benefit from that information, is nothing new. All social animals, and many that we generally consider to be nonsocial, have opportunities to observe the behaviour of members of their own or other species, and to use this information to modify their own behaviour (Danchin 2004). Social information is often created as an inadvertent by-product of behaviour, becoming a cue when the information reaches an observer. When social information is provided intentionally, this is called signalling. In insects, perhaps the most famous example of signalling is the symbolically transmitted information about food quality and location, encoded in the honey bee waggle dance. The benefits of producing and acquiring this information are obvious: successful foragers can recruit nestmates to abundant food resources, saving them the search costs of finding the resource independently. Ants provide another well-known example, with foragers laying down chemical trails that nestmates can follow.

Not all social insects have the ability to signal each other about foraging opportunities, but they do have the ability to acquire social information from cues produced by others. Considerable evidence demonstrates that bees both solitary and social, as well as other pollinators, including flies, use social information produced by other individuals of their own or different species. For instance, facultatively social carpenter bees chemically mark flowers that they have visited, which helps them to avoid re-visiting spent blossoms (Frankie and Vinson 1977). These chemical marks are public, so as long as other pollinators can "eavesdrop" and correctly perceive the cue, they too, can choose to avoid the spent flower.

M. H. Richards

mrichards@brocku.ca

1 Brock University, St. Catharines, Canada
Foraging stingless bees acquire information about the quality and location of food resources by learning, eavesdropping on cues provided by others, or from signals produced by nestmates. Foragers lay ephemeral but effective, airborne chemical trails for nestmates to follow to abundant food resources. They may also mark flowers. When bees land on surfaces, they leave tiny, chemical "footprints", either intentionally or inadvertently. Bees can deposit chemical products produced by foot or mandibular glands. Even if the bees themselves deposit no chemicals, a footprint can result as the plant reacts biochemically to being walked on (Bown et al. 2002). Regardless of how chemical signals and cues are produced, eavesdroppers may benefit by using the information.

In this issue, Rosalyn Gloag and colleagues (Gloag et al. 2021) investigate how publically available social information is produced and used by three species of Australian stingless bees. Stingless bees can be trained to forage at feeders, enabling Gloag et al. to use a classic, binary choice paradigm. Foraging stingless bees were first trained to visit feeders and then were offered a choice between a feeder that had been visited by other bees or a clean feeder that had not been visited. Assuming that foragers inadvertently or intentionally deposit chemicals when feeding, the feeder with previous foragers would provide social cues not available on the clean feeder. Stingless bees clearly preferred feeders previously visited by other bees. Thus the chemical footprints deposited functioned as attractants for subsequent foragers. For one species, it was even possible to estimate the chemical "dose" for the footprints to become attractive: for Trigonula carbonaria, it took about 20 bees to deposit enough odour to become an attractant. Intriguingly, the stingless bee foragers not only preferred feeders visited by nestmates, but also showed a preference for feeders visited by conspecifics from another colony, and even by foragers of another species, namely honeybees. The ability of the stingless bees to eavesdrop on the chemical cues of honey bees, a species introduced to Australia early in the nineteenth century, suggests that the attractant function traces back to the common ancestor of meliponine and apine bees. A reciprocal 
experiment testing whether honey bees are attracted to footprints of the stingless bees would help to address this.

For both solitary and social bees, marking flowers (or feeders) likely increases their own foraging efficiency, but also promotes the efficiency of competitors both conspecific and heterospecific, that can parasitize this information. How social information evolves likely reflects this kind of cost-benefit balance. My own impression is that many of the known chemical cues deposited by solitary bees are effectively self-repellents that help bees minimize the costs of wasted foraging effort (Yokoi and Fujisaki 2011). In contrast, in species like stingless bees, honey bees, and many ants, the known chemical foraging cues are attractants. Like all good science, Gloag et al.'s study raises many new and interesting questions: Do solitary bees ever mark flowers with attractant signals? Do species like stingless bees provide repellent, as well as attractant cues? Were there evolutionary switches from production of repellent to attractant cues that were associated with evolutionary transitions from solitary to social behaviour?

\section{References}

Bown AW, Hall DE, MacGregor KB (2002) Insect footsteps on leaves stimulate the accumulation of 4-aminobutyrate and can be visualized through increased chlorophyll fluorescence and superoxide production. Plant Physiol 129:1430-1434. https://doi.org/10.1104/ pp.006114

Danchin E (2004) Public information: from nosy neighbors to cultural evolution. Science 305:487-491. https://doi.org/10.1126/science. 1098254

Frankie GW, Vinson SB (1977) Scent marking of passion flowers in texas by females of Xylocopa virginica texana (Hymenoptera: Anthophoridae). J Kans Entomol Soc 50:613-625

Gloag R, Smith JP, Stephens RE et al (2021) Australian stingless bees detect odours left at food sources by nestmates, conspecifics and honey bees. Insect Soc. https://doi.org/10.1007/ s00040-021-00823-7

Yokoi T, Fujisaki K (2011) To forage or not: responses of bees to the presence of other bees on flowers. Ann Entomol Soc Am 104:353-357. https://doi.org/10.1603/AN10053 